\title{
N93-277338
}

\section{QUANTUM WORMHOLES}

\section{AND \\ HARMONIC OSCILLATORS}

\author{
Luis J. Garay \\ Instituto de Optica, Consejo Superior de Investigaciones Científicas, \\ Serrano 121, E-28006 Madrid, Spain
}

\begin{abstract}
The quantum state of a wormhole can be represented by a path integral over all asymptotically Euclidean four-geometries and all matter fields which have prescribed values, the arguments of the wave function, on a three-surface which divides the spacetime manifold into two disconnected parts. Minisuperspace models which consist of a homogeneous massless scalar field coupled to a Friedmann-Robertson-Walker spacetime are considered. Once the path integral over the lapse function is performed, the requirement that the spacetime be asymptotically Euclidean can be accomplished by fixing the asymptotic gravitational momentum in the remaining path integral. It is argued that there does not exist any wave function which corresponds to asymptotic field configurations such that the effective gravitational constant is negative in the asymptotic region. Then, the wormhole wave functions can be written as linear combinations of harmonic oscillator wave functions.
\end{abstract}

\section{Introduction}

Wormholes have been considered as instantons, solutions of the Euclidean Einstein equations, which consist of two asymptotically Euclidean regions connected by a throat $[1,2]$. These classical wormholes are saddle points of the Euclidean action and therefore, they allow the Euclidean path integral to be approximated semiclassically. One makes the dilute wormhole approximation in which the wormhole ends are far apart from each other, so that one can consider that wormholes do not interact and then, they can be treated separately. Wormholes on the Planck scale may affect the constants of nature and, in particular, may provide a mechanism for the vanishing of the cosmological constant $[3,4]$. Wormholes may play an important role in solving problems associated with the complete evaporation and disappearance of black holes [2]. However, classical wormholes may only exist for very special types of matter, those which allow the Ricci tensor to have negative eigenvalues $[1,5,6]$. This may place a strong restriction on the possibility that wormholes have any role in these processes.

However, we need not restrict ourselves to such a semiclassical treatment and the special types of matter that it requires. More generally, one can regard wormholes as solutions of the quantum Wheeler-DeWitt equation with some suitable boundary conditions. Hawking and Page [7] have 
proposed that the boundary conditions are as follows. The wormhole wave function can be represented as the path integral over all asymptotically Euclidean four-geometries that match a given compact three-geometry which is the argument of the wave function. This can be interpreted as saying that there are no gravitational excitations at infinity (i.e., at large distance compared with the characteristic scale of the wormhole). An extra surface term which eliminates the infinite contribution that comes from the asymptotically Euclidean region should be added to the action. It will be seen that, once the path integrals over the lapse and shift functions have been performed, the requirement that the spacetime is asymptotically Euclidean is equivalent to the fixation of the asymptotic gravitational momentum [8]. Also, one must integrate over all matter fields which have no sources in the asymptotic region. This reflects the fact that there are no matter excitations at infinity. This is accomplished by requiring that the matter Hamiltonian must vanish asymptotically. If one considers a coupled scalar field with a potential as the matter content, this means that, at infinity, the field must approach an homogeneous configuration at which the potential has a vanishing minimum [8].

In what follows, we shall study a massless homogeneous scalar field minimally and conformally coupled to a Friedmann-Robertson-Walker (FRW) spacetime. It turns out that, as in many other physical theories, harmonic oscillators are present in wormhole physics. In fact the wormhole wave functions, in these models can be written in terms of harmonic oscillators wave functions.

\section{Minimal scalar field}

We shall consider a massless homogeneous scalar field $\phi(\tau)$ minimally coupled to a FRW whose metric can be written

$$
d s^{2}=\frac{2 \mathrm{G}}{3 \pi}\left(\frac{N^{2}(\tau)}{2 q(\tau)} d \tau^{2}+2 q(\tau) d \Omega_{3}^{2}\right)
$$

where $N(\tau)$ is the lapse function which measures the proper time separation between two neighbouring three-spheres whose radii are represented by $\sqrt{2 q(\tau)}$. The action for this system takes the simple form

$$
\tilde{I}=\int_{0}^{\infty} d \tau\left(\pi_{q} \dot{q} \pi_{\phi} \dot{\phi}-N H\right), \quad H=\frac{1}{2}\left(-\pi_{q}^{2}+1\right)+\frac{1}{8} q^{-2} \pi_{\phi}^{2},
$$

whre $H$ is the Hamiltonian and the relations between the canonical momenta $\pi_{q}, \pi_{\phi}$, and the time derivatives of the variables are given by $\pi_{q}=-\dot{q} / N, \pi_{\phi}=q^{2} \dot{\phi} / 4 N$. The wormhole wave function is defined by the path integral over all asymptotically Euclidean four-metrics and over all scalar fields whose asymptotic configuration is given by a constant value $\phi_{0}$. If the four-metric is going to be asymptotically Euclidean, then the variable $q$ must have the behaviour $q(\tau) \sim \int d \tau N(\tau)$ when $\tau \rightarrow \infty$, as can be seen from the expression for the line element. This means that the asymptotic condition must be $\pi_{q}(\infty)=-1$. Since we have to fix the asymptotic momentum rather than the canonical variable $q$, it will be necessary to introduce the term $\left.\pi_{q} q\right|_{\tau=\infty}$ in the action, so that the variational problem associated to it, subjected to the boundary conditions mentioned above, be well posed. Therefore, the action will have the form $I=\tilde{I}-\left.\pi_{q} q\right|_{r=\infty}$. 
Under time reparametrizations defined by the transformations

$$
\delta N(\tau)=\dot{\epsilon}(\tau), \quad \delta \zeta=\{\zeta, \epsilon H\}, \quad \delta \pi_{\zeta}=\left\{\pi_{\zeta}, \epsilon H\right\}, \quad(\zeta=q, \phi)
$$

the variation of the action is $\delta I=-\epsilon(0)\left[\sum_{\zeta=q, \phi} \pi_{\zeta}\{\zeta, H\}-H\right]_{\tau=0}+\left.\frac{1}{2} \epsilon(\infty) H\right|_{\tau=\infty}$. The boundary conditions ensure that $\left.H\right|_{\tau=0}=0$ and, therefore, the action will be invariant under transformations such that $\epsilon(0)=0$, i.e., that do not change the arguments of the wave function, which are defined at $\tau=0: \quad q(0)=q^{\prime}, \phi(0)=\phi^{\prime}$. The wave function can, then, be written as

$$
\Psi\left(q^{\prime}, \phi^{\prime}\right)=\int \mathcal{D} N \int_{\mathcal{C}_{\text {wh }}} \prod_{\zeta=q, \phi} \mathcal{D} \zeta \mathcal{D} \pi_{\zeta} \delta(F) \Delta_{F P} e^{-I\left[\zeta, \pi_{\zeta}, N\right]},
$$

where $\mathcal{C}_{w h}$ is the set of histories which satisfy the boundary conditions, $F$ is the gauge fixing condition that singles out a representant of each equivalence class under time reparametrizations and $\Delta_{F P}$ is the Fadeev-Popov determinant which ensures that the path integral does not depend on this choice. It is easily seen that the simplest admissible [9] gauge fixing condition is $N=1$. Any other history $N(\tau)$ can be obtained from this one by means of the time reparametrization whose coefficient $\epsilon(\tau)$ is the only solution to the equation $N(\tau)-1=\dot{\epsilon}(\tau)$, subjected to the condition $\epsilon(0)=0$. This solution is $\epsilon(\tau)=-\tau+\int_{0}^{\tau} d \tau N(\tau)$. The Fadeev-Popov determinant is independent of the integration variables and, therefore, the wave function acquires the form [8]

$$
\Psi\left(q^{\prime}, \phi^{\prime}\right)=\int_{\mathcal{C}_{w \mathrm{~b}}} \mathcal{D} q \mathcal{D} \pi_{q} \mathcal{D} \phi \mathcal{D} \pi_{\phi} e^{-I[q, \phi]}
$$

where $I[q, \phi]=\int_{0}^{\infty} d \tau\left(\pi_{q} \dot{q}+\pi_{\phi} \dot{\phi}-H\right)-\left.q \pi_{q}\right|_{\tau=\infty}$, and $\mathcal{C}_{\text {wh }}$ is the set of histories such that

$$
q(0)=q^{\prime}, \quad \phi(0)=\phi^{\prime}, \quad \pi_{q}(\infty)=-1, \quad \phi(\infty)=\phi_{0} .
$$

With the change of variables [10]

$$
q=\left(x^{2}-t^{2}\right)^{\frac{1}{2}}, \quad \phi=\frac{1}{2} \tanh ^{-1} \frac{t}{x}, \quad q \pi_{q}=x \pi_{x}+t \pi_{t}, \quad \pi_{\phi}=2\left(x \pi_{t}+t \pi_{x}\right),
$$

the action takes the simple form

$$
I=\int_{0}^{\infty} d \tau\left(\pi_{x} \dot{x}+\pi_{t} \dot{t}-H\right)-\left.\left(x \pi_{x}+t \pi_{t}\right)\right|_{\tau=\infty}, \quad H=\frac{1}{2}\left(-\pi_{x}^{2}+\pi_{t}^{2}+1\right) .
$$

The boundary conditions (6) transform into

$$
x(0)=q^{\prime} \cosh 2 \phi^{\prime}, \quad t(0)=q^{\prime} \sinh 2 \phi^{\prime}, \quad \pi_{x}(\infty)=-\cosh 2 \phi_{0}, \quad \pi_{t}(\infty)=\sinh 2 \phi_{0} .
$$

and the measure into $\mathcal{D} x \mathcal{D} t \mathcal{D} \pi_{x} \mathcal{D} \pi_{t}$, because the Jacobian is equal te one.

To do the path integral

$$
\Psi_{\phi_{0}}\left(q^{\prime}, \phi^{\prime}\right)=\int \mathcal{D} x \mathcal{D} t \mathcal{D} \pi_{x} \mathcal{D} \pi_{t} e^{-I[x, t]}
$$


with the boundary conditions (9), it is convenient to shift the integration variables

$$
x(\tau)=\bar{x}(\tau)+X(\tau), \quad t(\tau)=\bar{t}(\tau)+T(\tau),
$$

where $\bar{x}$ and $\bar{t}$ correspond to the classical solution of the variational problem associated with (8) which satisfies the boundary conditions (9). Explicitly, this solution is

$$
\bar{x}(\tau)=\tau \cosh 2 \phi_{0}+q^{\prime} \cosh 2 \phi^{\prime}, \quad \bar{t}(\tau)=\tau \sinh 2 \phi_{0}+q^{\prime} \sinh 2 \phi^{\prime} .
$$

The new variables $X$ and $T$ satisfy the conditions: $X(0)=T(0)=0, \quad P_{X}(\infty)=P_{T}(\infty)=0$. Then, the action (8) splits into two parts

$$
I[x, t]=I_{0}[\bar{x}, \bar{t}]+I_{2}[X, T],
$$

where $I_{0}[\bar{x}, \bar{t}]=q^{\prime} \cosh \left(\phi^{\prime}-\phi_{0}\right)$ is the action for the classical solution (12) and

$$
I_{2}[X, T]=\int_{0}^{\infty} d \tau\left\{P_{X} \dot{X}+P_{T} \dot{T}-\frac{1}{2}\left(-P_{X}^{2}+P_{T}^{2}\right)\right\} .
$$

The linear term $I_{1}[\bar{x}, \bar{t}, X, T]$ vanishes identically due to the fact that (12) is a saddle point of the action (8). The measure in the path integral (10) is directly changed into $\mathcal{D} X \mathcal{D} P_{X} \mathcal{D} T \mathcal{D} P_{T}$. $I_{0}$ does not depend on $X$ or $T$ and then, can be taken out of the integral. The remaining path integral becomes $\int \mathcal{D} X \mathcal{D} T \exp \left\{-\frac{1}{2} \int_{0}^{\infty} d \tau\left(-\dot{X}^{2}+\dot{T}^{2}\right)\right\}$ which is independent of $q^{\prime}$ and $\phi^{\prime}$ and therefore, it turns out to be a numerical factor [10]. Thus, up to numerical prefactors,

$$
\Psi_{\phi_{0}}(q, \phi)=\exp \left\{-q \cosh 2\left(\phi-\phi_{0}\right)\right\}
$$

which had already been found as a solution of the Wheeler-DeWitt equation [11]. This wave function can also be written as a linear combination of harmonic oscillator wave functions

$$
\Psi_{\phi_{0}}(q, \phi)=\sum_{n=0}^{\infty} c_{n}\left(\phi_{0}\right) \Psi_{n}(q, \phi), \quad c_{n}\left(\phi_{0}\right)=\frac{\sinh ^{n} \phi_{0}}{\cosh ^{n+1} \phi_{0}}
$$

where $\Psi_{n}(q, \phi)=\psi_{n}(\sqrt{2 q} \cosh \phi) \psi_{n}(\sqrt{2 q} \sinh \phi)$ and $\psi_{n}(x)$ are the harmonic oscillator eigenfunctions.

This wave function behaves in a regular way when the three-geometry degenerates. However, the linear combination

$$
\Psi_{k}(q, \phi)=\int_{-\infty}^{+\infty} d \phi_{0} e^{-i k \phi_{0}} \Psi_{\phi_{0}}(q, \phi)
$$

is an eigenfunction of the operator $\pi_{\phi}$ with eigenvalue $k$ and therefore, has non zero flux through each three-surface. This wave function cannot close off with a four-geometry and will oscillate an infinite number of times when the three-geometry collapses to zero. In fact, once the integral over $\phi_{0}$ is performed, the wave function (17) takes the form

$$
\Psi_{k}(q, \phi)=K_{i \frac{k}{2}}(q) e^{-i k \phi}
$$


where $K_{i k / 2}(q)$ is a modified Bessel function of imaginary order. This wave function will oscillate for $q<|k / 2|$, while for $q>|k / 2|$, it will decrease exponentially. Thus, $|k / 2|$ can be considered as the throat radius of the wormhole [7]. Since (17) is a Fourier transform, it suggests a kind of uncertainty relation between the asymptotic field and the wormhole throat radius. This could be expected because $k$ does not only represent the throat size but it is also the eigenvalue of the momentum conjugate to the scalar field and therefore, $k$ and $\phi_{0}$ naturally satisfy an uncertainty relation [11].

\section{Conformal scalar field}

When an homogeneous scalar field $\varphi(\tau)$ is conformally coupled to a FRW spacetime, the path integral which defines the wormhole wave function can also be done explicitly in a similar way. The FRW metric can be written in the convenient form

$$
d s^{2}=\frac{2 \mathrm{G}}{3 \pi} a^{2}(\tau)\left(d \tau^{2}+d \Omega_{3}^{2}\right)
$$

With the field redefinition $\varphi(\tau)=\chi(\tau) a^{-1}(\tau)$, the Euclidean action for this system becomes

$$
I=\frac{1}{2} \int_{0}^{\infty} d \tau\left(-\dot{a}^{2}-a^{2}+\dot{\chi}^{2}+\chi^{2}\right)+\left.\frac{1}{2} \frac{\dot{a}}{a}\left(a^{2}-\chi^{2}\right)\right|_{\tau=\infty} .
$$

The boundary conditions are, in this case,

$$
a(0)=a^{\prime}, \quad \varphi(0)=\varphi^{\prime}=\frac{\chi^{\prime}}{a^{\prime}}, \quad \frac{\dot{a}}{a}(\infty)=1, \quad \varphi(\infty)=\frac{\chi}{a}(\infty)=\varphi_{0}
$$

The conditions at $\tau=0$ indicate which are the arguments of the wave function. The conditions at $\tau \rightarrow \infty$ mean that the spacetime is asymptotically Euclidean and that the field $\varphi$ takes the value $\varphi_{0}$ at infinity. The wormhole wave function will be labeled by the asymptotic field value $\varphi_{0}$ :

$$
\Psi_{\varphi_{0}}\left(a^{\prime}, \chi^{\prime}\right)=\int_{\mathcal{C}_{w h}} \mathcal{D} a \mathcal{D} \chi e^{-I[a, \chi]},
$$

where $\mathcal{C}_{\text {wh }}$ is the class of histories that satisfy (21).

Let

$$
\begin{aligned}
& \bar{a}(\tau)=a^{\prime} \cosh \tau+\frac{1+\varphi_{0}^{2}}{1-\varphi_{0}^{2}}\left(a^{\prime}-\frac{2 \varphi_{0}}{1+\varphi_{0}^{2}} \chi^{\prime}\right) \sinh \tau, \\
& \bar{\chi}(\tau)=\chi^{\prime} \cosh \tau+\frac{1+\varphi_{0}^{2}}{1-\varphi_{0}^{2}}\left(\frac{2 \varphi_{0}}{1+\varphi_{0}^{2}} a^{\prime}-\chi^{\prime}\right) \sinh \tau
\end{aligned}
$$

be the solution of the classical equations of motion that satisfies (21). Then, as in the case of a minimal coupling and due to the fact that the action is also quadratic, under the shift of the integration variables $a=\bar{a}+A, \quad \chi=\bar{\chi}+X$, where the new variables $A$ and $X$ are such that

$$
A(0)=X(0)=0, \quad \frac{\dot{A}}{A}(\infty)=1, \quad \frac{X}{A}(\infty)=\varphi_{0},
$$


the path integral (22) transforms into

$$
\Psi_{\varphi_{0}}\left(a^{\prime}, \chi^{\prime}\right)=e^{-I_{0}\left(a^{\prime}, \chi^{\prime}\right)} \int \mathcal{D} A \mathcal{D} X e^{-I_{2}[A, X]},
$$

where $I_{2}[A, X]$ does not depend on $a^{\prime}$ or $\chi^{\prime}$ and $I_{0}\left(a^{\prime}, \chi^{\prime}\right)$ is the action of the classical solution (23). Then, up to numerical factors,

$$
\Psi_{\varphi_{0}}(a, \chi)=\exp \left\{-\frac{1}{2}\left(\frac{1+\varphi_{0}^{2}}{1-\varphi_{0}^{2}}\right)\left(a^{2}+\chi^{2}-\frac{4 \varphi_{0}}{1+\varphi_{0}^{2}} a \chi\right)\right\} .
$$

As it was expected, this wave function is also a solution of the Wheeler-DeWitt equation. The condition

$$
\varphi_{0}^{2}<1
$$

must be fulfilled by the asymptotic field in order to have a positive effective gravitational constant in the asymptotic region [8]. Then, (26) will represent the wormhole wave function. Condition (27) will also allow us to write this wave function as a linear combination of those obtained in [2] and [7], as it happens in the previous section:

$$
\Psi_{\varphi_{0}}(a, \chi)=\sum_{n=0}^{\infty} \varphi_{0}^{n}\left(1-\varphi_{0}^{2}\right)^{\frac{1}{2}} \Psi_{n}(a, \chi),
$$

where $\Psi_{n}(a, \chi)=\psi_{n}(a) \psi_{n}(\chi)$

\section{Summary and conclusions}

In the context of the minisuperspace models which consist of a homogeneous massless scalar field minimally and conformally coupled to a FRW spacetime, it has been performed the path integral that defines the wormhole wave function. The wormhole boundary conditions require that the spacetime be asymptotically Euclidean. This implies that the asymptotic gravitational momentum must have a given value at infinity. Therefore, a surface term which takes this fact into account must be added to the action. The path integral over the lapse function can be interpreted as a sum over all proper time separations between the asymptotic region and the surface in which the arguments of the wave function are defined. This separation is infinite and, therefore, the whole sum is trivially reduced to a single contribution. The action is quadratic and this allowed us to perform the whole path integral. The problem of the unboundedness from below of the action is harmless in these models, since the unbounded part can be isolated in a term which does not depend on the arguments of the wave function and can, therefore, be factored out of the wave function. In the case of a conformal scalar field, one must also impose the condition that the asymptotic gravitational constant be positive. When this physical requirement is satisfied, the wormhole wave functions can be written as linear combinations -of harmonic oscillator wave functions. 


\section{Acknowledgements}

I am very grateful to Pedro González-Díaz for many enlightening discussions and encouraging comments. Also, I wish to thank Guillermo Mena Marugán for valuable explanations and Jonathan Halliwell for useful conversations and for introducing me to some references relevant to this work.

\section{References}

[1] S. B. Giddings and A. Strominger, Nucl. Phys. B306, 890 (1988)

[2] S. W. Hawking, Phys. Rev. D37, 904 (1988)

[3] S. Coleman, Nucl. Phys. B310, 643 (1988)

[4] S. W. Hawking, Nucl. Phys. B335, 155 (1990)

[5] S. Bochner, Bull. Am. Math. Soc. 52, 776 (1946)

[6] J. Cheeger and D. Gromoll, Ann. Math. 96, 413 (1972)

[7] S. W. Hawking and D. N. Page, Phys. Rev. D42, 2655 (1990)

[8] L. J. Garay, Phys. Rev. D44, 1059 (1991)

[9] C. Teitelboim, Phys. Rev. D28, 297 (1983)

[10] L. J. Garay, J. J. Halliwell and G. A. Mena Marugán, Phys. Rev. D43, 2572 (1991)

[11] L. M. Campbell and L. J. Garay, Phys. Lett. B254, 49 (1991) 
